\title{
Validation of the Publication of New Names and New Combinations Previously Effectively Published Outside the IJSB
}

\author{
List No. $41 \dagger$
}

The purpose of this announcement is to effect the valid publicaiton of the following new names and new combinations under the procedure described previously [Int. J. Syst. Bacteriol. 27(3):iv, 1977]. Authors and other individuals wishing to have new names and/or combinations included in future lists should send the pertinent reprint or a photocopy thereof to the IJSB (c/o ASM) for confirmation that all of the other requirements for valid publication have been met. It should be noted that the date of valid publication of these new names and combinations is the date of publication of this list, not the date of the original publicaiton of the names and combinations. The authors of the new names and combinations are as given below, and these authors' names will be included in the author index of the present issue and in the volume author index in this issue of the IJSB.

Inclusion of a name on these lists validates the name and thereby makes it available in bacteriological nomenclature. The inclusion of a name on this list is not to be construed as taxonomic acceptance of the taxon to which the name is applied. Indeed, some of these names may, in time, be shown to be synonyms, or the organisms may be transferred to another genus, thus necessitating the creation of a new combination.

\begin{tabular}{lllcl}
\hline \multicolumn{1}{c}{ Name } & Proposed as: & \multicolumn{1}{c}{ Author(s) (reference) } & Priority $^{a}$ & \multicolumn{1}{c}{ Nomenclatural type $^{b}$} \\
\hline $\begin{array}{l}\text { Afipia } \\
\text { Afipia broomeae }\end{array}$ & New genus & Brenner et al. (3) & 3 & Afipia felis \\
Afipia clevelandensis & New species & Brenner et al. (3) & 3 & Strain B-91-007286 (= ATCC 49717) \\
Afipia felis & New species & Brenner et al. (3) & 3 & Strain B-91-007353 (= ATCC 49720) \\
& New species & Brenner et al. (3) & 3 & $\begin{array}{c}\text { Strain B-91-007352 = AFIP strain BV } \\
\text { (= ATCC 53690) }\end{array}$ \\
Bifidobacterium saeculare & New species & Biavati et al. (2) & 8 & Strain RA161 (= ATCC 49392) \\
Clostridium termitidis & New species & Hethener et al. (4) & 7 & Strain CT1112 (= DSM 5398) \\
Ehrlichia chaffeensis & New species & Anderson et al. (1) & 4 & Strain Arkansas (= ATCC CRL 10679) \\
Flavobacteriaceae & New family & Reichenbach (9) & 5 & Flavobacterium \\
Halobacteroides lacunaris & New species & Zhilina et al. (12) & 2 & Strain Z-7888 (= DSM 6640) \\
Kitasatosporia cystarginea & New species & Kusakabe and Isono (6) & 6 & Strain RK-419 (= JCM 7356 = FERM P-8006) \\
Methanopyrus & New genus & Kurr et al. (5) & 2 & Methanopyrus kandleri \\
Methanopyrus kandleri & New species & Kurr et al. (5) & 2 & Strain AV19 (= DSM 6324) \\
Pasteuria nishizawae & New species & Sayre et al. (10) & 1 & This organism has not been cultivated; the \\
& & & & type descriptive material consists of the de- \\
& & & & scriptions and illustrations in the paper by \\
Propionibacterium innocuum & New species & Pitcher and Collins (8) & 9 & Strain L60 (= NCTC 11082) \\
Thermotoga thermarum & New species & Windberger et al. (11) & 2 & Strain LA3 (= DSM 5069) \\
Trabulsiella & New genus & McWhorter et al. (7) & 1 & Trabulsiella guamensis \\
Trabulsiella guamensis & New species & McWhorter et al. (7) & 1 & Strain CDC 0370-85 (= ATCC 49490) \\
\hline
\end{tabular}

$\dagger$ Lists 1 through 40 were published in the Int. J. Syst. Bacteriol. 27:306, 1977; 29:79, 436, 1979; 30:601, 676, 1980; 31:215, 382, 1981; 32:266, 384, 1982; 33:438, $672,896,1983 ; \mathbf{3 4}: 91,270,355,503,1984 ; \mathbf{3 5}: 223,375,535,1985 ; \mathbf{3 6}: 354,489,573,1986 ; 37: 179,1987 ; 38: 136,220,328,449 ; 1988 ; 39: 93,205,371,495,1989 ; 40: 105$, $212,320,470,1990 ; 41: 178,331,456,580,1991 ;$ and 42:191, 1992.

${ }^{a}$ Priority number assigned according to the date the documentation and request for validation are received.

${ }^{b}$ Abbreviations: ATCC, American Type Culture Collection, Rockville, Md.; DSM, Deutsche Sammlung von Mikroorganismen und Zellkulturen GmbH, Braunschweig, Germany; FERM, Fermentation Research Institute, Ibaraki, Japan; JCM, Japan Collection of Microorganisms, Riken, Saitama, Japan; NCTC, National Collection of Type Cultures, London, England.

\section{REFERENCES}

1. Anderson, B. E., J. E. Dawson, D. C. Jones, and K. H. Wilson. 1991. Ehrlichia chaffeensis, a new species associated with human ehrlichiosis. J. Clin. Microbiol. 29:2838-2842.

2. Biavati, B., P. Mattarelli, and F. Crociani. 1991. Bifidobacterium saeculare: a new species isolated from feces of rabbit. Syst. Appl. Microbiol. 14:389-392.

3. Brenner, D. J., D. G. Hollis, C. W. Moss, C. K. English, G. S. Hall, J. Vincent, J. Radosevic, K. A. Birkness, W. F. Bibb, F. D. Quinn, B. Swaminathan, R. E. Weaver, M. W. Reeves, S. P. O'Connor, P. S. Hayes, F. C. Tenover, A. G. Steigerwalt, B. A. Perkins, M. I. Daneshvar, B. C. Hill, J. A. Washington, T. C. Woods, S. B. Hunter, T. L. Hadfield, G. W. Ajello, A. F. Kaufmann, D. J. Wear, and J. D. Wenger. 1991. Proposal of Afipia gen. nov., with Afipia felis sp. nov. (formerly the cat scratch disease bacillus), Afipia clevelandensis sp. nov. (formerly the Cleveland Clinic Foundation strain), Afipia broomeae sp. nov., and three unnamed genospecies. J. Clin. Microbiol. 29:2450-2460.

4. Hethener, P., A. Brauman, and J.-L. Garcia. 1992. Clostridium termitidis sp. nov., a cellulolytic bacterium from the gut of the wood-feeding termite, Nasutitermes lujae. Syst. Appl. Microbiol. 15:52-58.

5. Kurr, M., R. Huber, H. König, H. W. Jannasch, H. Fricke, A. Trincone, J. K. Kristjansson, and K. O. Stetter. 1991. Methanopyrus kandleri, gen. and sp. nov. represents a novel group of hyperthermophilic methanogens, growing at $110^{\circ} \mathrm{C}$. Arch. Microbiol. 156:239-247.

6. Kusakabe, H., and K. Isono. 1988. Taxonomic studies on 
Kitasatosporia cystarginea sp. nov., which produces a new antifungal antibiotic cystargin. J. Antibiot. 41:1758-1762.

7. McWhorter, A. C., R. L. Haddock, F. A. Nocon, A. G. Steigerwalt, D. J. Brenner, S. Aleksic, J. Bockemühl, and J. J. Farmer III. 1991. Trabulsiella guamensis, a new genus and species of the family Enterobacteriaceae that resembles Salmonella subgroups 4 and 5. J. Clin. Microbiol. 29:1480-1485.

8. Pitcher, D. G., and M. D. Collins. 1991. Phylogenetic analysis of some LL-diaminopimelic acid-containing coryneform bacteria from human skin: description of Propionibacterium innocuum sp. nov. FEMS Microbiol. Lett. 84:295-300.

9. Reichenbach, H. 1989. Order 1. Cytophagales Leadbetter 1974, 99AL, p. 2011-2013. In J. T. Staley, M. P. Bryant, N. Pfennig, and J. G. Holt (ed.), Bergey's manual of systematic bacteriol- ogy, vol. 3. The Williams \& Wilkins Co., Baltimore.

10. Sayre, R. M., W. P. Wergin, J. M. Schmidt, and M. P. Starr. 1991. Pasteuria nishizawae sp. nov., a mycelial and endosporeforming bacterium parasitic on cyst nematodes of genera Heterodera and Globodera. Res. Microbiol. 142:551-564.

11. Windberger, E., R. Huber, A. Trincone, H. Fricke, and K. $O$. Stetter. 1989. Thermotoga thermarum sp. nov. and Thermotoga neapolitana occurring in African continental solfataric springs. Arch. Microbiol. 151:506-512.

12. Zhilina, T. N., L. V. Miroshnikova, G. A. Osipov, and G. A. Zavarzin. 1991. Halobacteroides lacunaris sp. nov.-a new saccharolytic anaerobic extremely halophilic organism from lagoonic hypersaline Lake Chokrak. Mikrobiologiya 60:714 724.

\section{Erratum}

\section{List No. 37 (IJSB 41:331, 1991)}

Propionivibrio, a new genus with the type species Propionivibrio dicarboxylicus, was inadvertently omitted (authors, Tanaka et al.; priority, 2). The effective date of validation is that of list no. 37.

Notification that New Names and New Combinations Have Appeared in the IJSB ${ }^{a}$

\begin{tabular}{|c|c|c|c|}
\hline Name & Proposed as: & Author(s) & $\begin{array}{l}\text { IJSB reference } \\
\text { description }\end{array}$ \\
\hline $\begin{array}{l}\text { Helicobacter muridarum } \\
\text { Prolinoborus } \\
\text { Prolinoborus fasciculus (basonym: Aquaspirillum } \\
\quad \text { fasciculus) }\end{array}$ & $\begin{array}{l}\text { sp. nov. } \\
\text { gen. nov. } \\
\text { comb. nov. }\end{array}$ & $\begin{array}{l}\text { Lee et al. } \\
\text { Pot et al. } \\
\text { Pot et al. }\end{array}$ & $\begin{array}{l}42(1): 35 \\
42(1): 52 \\
42(1): 53\end{array}$ \\
\hline Clavibacter toxicus & sp. nov. & Riley and Ophel & 42(1):67 \\
\hline Listeria ivanovii subsp. londoniensis & subsp. nov. & Boerlin et al. & $42(1): 73$ \\
\hline Brachybacterium nesterenkovii & sp. nov. & Gvozdyak et al. & 42(1):77 \\
\hline Alloiococcus & gen. nov. & Aguirre and Collins & $42(1): 83$ \\
\hline Alloiococcus otitis & sp. nov. & Aguirre and Collins & 42(1):83 \\
\hline Aminobacter & gen. nov. & Urakami et al. & 42(1):90 \\
\hline $\begin{array}{l}\text { Aminobacter aminovorans (basonym: Pseudomonas } \\
\text { aminovorans) }\end{array}$ & comb. nov. & Urakami et al. & $42(1): 90$ \\
\hline Aminobacter aganoensis & sp. nov. & Urakami et al. & 42(1):91 \\
\hline Aminobacter niigataensis & sp. nov. & Urakami et al. & 42(1):91 \\
\hline Staphylococcus muscae & sp. nov. & Hájek et al. & 42(1):99 \\
\hline Sphingobacterium antarcticus & sp. nov. & Shivaji et al. & 42(1): 105 \\
\hline Acidovorax avenae & comb. nov. & Willems et al. & 42(1):115 \\
\hline $\begin{array}{l}\text { Acidovorax avenae subsp. avenae (basonym: } \\
\text { Pseudomonas avenae) }\end{array}$ & subsp. nov., comb. nov. & Willems et al & 42(1):117 \\
\hline $\begin{array}{l}\text { Acidovorax avenae subsp. cattleyae (basonym: } \\
\text { Pseudomonas cattleyae) }\end{array}$ & comb. nov. & Willems et al. & $42(1): 118$ \\
\hline $\begin{array}{l}\text { Acidovirax avenae subsp. citrulli (basonym: } \\
\text { Pseudomonas pseudoalcaligenes subsp. citrulli) }\end{array}$ & comb. nov. & Willems et al. & $42(1): 118$ \\
\hline $\begin{array}{l}\text { Acidovorax konjaci (basonym: Pseudomonas } \\
\text { pseudoalcaligenes subsp. konjaci) }\end{array}$ & comb. nov. & Willems et al. & 42(1):118 \\
\hline Piscirickettsia & gen. nov. & Fryer et al. & $42(1): 123$ \\
\hline Piscinickettsia salmonis & sp. nov. & Fryer et al. & 42(1): 123 \\
\hline Agrobacterium atlanticum & sp. nov. & Rüger and Höfle & 42(1):141 \\
\hline Agrobacterium meteori & sp. nov. & Rüger and Höfle & 42(1):141 \\
\hline Agrobacterium ferrugineum & sp. nov., nom. rev. & Rüger and Höfle & 42(1):141 \\
\hline Agrobacterium gelatinovorum & sp. nov., nom. rev. & Rüger and Höfle & 42(1): 141 \\
\hline Agrobacterium stellulatum & sp. nov., nom. rev. & Rüger and Höfle & 42(1):141 \\
\hline Actinomyces suis (basonym: Eubacterium suis) & comb. nov. & Ludwig et al. & 42(1): 164 \\
\hline Spirochaeta thermophila & sp. nov. & Aksenova et al. & 42(1):176 \\
\hline Corynebacterium urealyticum & sp. nov. & Pitcher et al. & 42(1): 180 \\
\hline Cytophaga marina pro. synon. Flexibacter maritimus & synon. & Holmes & $42(1): 185$ \\
\hline
\end{tabular}

